ISSN 1991-8631

Original Paper

http://indexmedicus.afro.who.int

\title{
Yield loss of Oryza glaberrima caused by grain shattering under rainfed upland conditions
}

\author{
D. MONTCHO ${ }^{1,2, *}$, K. FUTAKUCHI $^{1}$, C. AGBANGLA ${ }^{2}$, M. FOFANA ${ }^{3}$ and I. DIENG ${ }^{1}$ \\ ${ }^{1}$ Africa Rice Center (AfricaRice), 01 BP 2031 Cotonou, Benin. \\ ${ }^{2}$ University of Abomey-Calavi, BP 526 Abomey-Calavi, Benin. \\ ${ }^{3}$ Africa Rice Center (AfricaRice), PMB 5320, Ibadan, Nigeria. \\ * Corresponding author, E-mail: montchodav@yahoo.fr; Tel: +229-95767060
}

\begin{abstract}
The general perception of Oryza glaberrima as low yielding is a potential drawback to intraspecific breeding of this species. Yields of 21 O. glaberrima accessions were evaluated alongside four $O$. sativa check varieties under two conditions in which panicles were both covered by paper sacks to avoid grain shattering (potential yield plot) and also not covered (control plot) in a rainfed upland field of Benin in 2010 and 2011. Average potential yields of $O$. glaberrima with panicles covered were 2.42 and $3.65 \mathrm{t} \mathrm{ha}^{-1}$ in 2010 and 2011, respectively, while those of $O$. sativa were respectively 2.99 and $3.49 \mathrm{t} \mathrm{ha}^{-1}$. Although there was no significant difference between potential yield and control yield in O. sativa, 19 O. glaberrima accessions showed significant yield reduction by grain shattering - the average percentages of lost yield by shattering were $57.88 \%$ in 2010 and $66.76 \%$ in 2011 . These results suggest that low yield of $O$. glaberrima is due to grain shattering and that its potential yield would not be inferior to $O$. sativa if the grain shattering problems were overcome. Two $O$. glaberrima accessions, TOG 12303 and TOG 9300, possessing strong resistance to grain shattering comparable to $O$. sativa checks, were identified.
\end{abstract}

() 2013 International Formulae Group. All rights reserved.

Keywords: Panicles, paper sacks, potential yield, yield reduction.

\section{INTRODUCTION}

Only two species - Oryza glaberrima Steud. and Oryza sativa L.- within the genus Oryza comprising 22 species were cultivated (Vaughan et al., 2003). Oryza glaberrima, the indigenous African rice, is thought to have been domesticated between 2000 and 3000 years ago in the inland delta of the upper Niger river in West Africa. This domestication taking place long before the introduction of $O$. sativa to the continent by the Portuguese (Linares, 2002). Because of the history of $O$. glaberrima in Africa, the species is expected to be a rich genetic source for adaptability to African environments as compared to $O$. sativa. In fact $O$. glaberrima can provide resistance to various indigenous biotic constraints: rice yellow mottle virus (Thiémélé et al., 2010), bacterial leaf blight (Djedatin et al., 2011), African rice gall midge (Ukwungwu et al., 1999; Williams et al., 1999), weeds (Moukoumbi et al., 2011) and nematode (Coyne et al., 1999; PlowRight et al., 1999) and to abiotic stresses such as drought (Maji et al., 2010; Ndjiondjop et al., 2010), submergence (Futakuchi et al., 2001; 
Kawano et al., 2008), iron toxicity (Sahrawat and Sika, 2002) and low phosphorus availability in acid soils (Tobita et al., 2003). Interspecific breeding of $O$. glaberrima with high yielding $O$. sativa could be one approach to use the advantage (resistance to local constraints) of $O$. glaberrima while excluding its low yield characteristic (Ghesquiere et al., 1997) and to create a number of interspecific lines both for upland (Jones et al., 1997) and lowland (Sie et al., 2005). To develop the fertile interspecific lines, two to four backcrosses to the $O$. sativa were needed (Jones et al., 1997; Sie et al., 2005). Intraspecific $O$. glaberrima $\times O$. glaberrima breeding, which could be another approach to better utilize the values of $O$. glaberrima for farmers in Africa (Futakuchi and Sie, 2009). Oryza glaberrima was previously considered as low yield (Linares, 2002) and actually shown to have negative characteristics for yield generation such as grain shattering (Jones et al., 1997) and lodging (Futakuchi et al., 2008). Dingkuhn et al. (1998) measured number of spikelets before the occurrence of shattering for an $O$. glaberrima accession (CG 14 ) and $O$. sativa and interspecific varieties in upland with several nitrogen application levels, and showed that the number of spikelets per unit ground area was not low in O. glaberrima compared to $O$. sativa and interspecific varieties, suggesting low yield of $O$. glaberrima could be due to grain shattering and its yield potential is not inferior to $O$. sativa. To assure the prospects for intraspecific breeding, more information on the relationship between grain shattering and yield using a larger number of $O$. glaberrima accessions is necessary.

Therefore, the objectives of this study were: to evaluate the yield of $O$. glaberrima accessions if no incidence of grain shattering occurs (hereafter we call it potential yield); to know the range of yield loss from grain shattering within $O$. glaberrima (evaluation for the varietal difference of yield loss); and to identify $O$. glaberrima accessions which show resistance to grain shattering.

\section{MATERIALS AND METHODS}

The trial was conducted in 2010 and 2011 under upland conditions at the AfricaRice (Africa Rice Center) experimental site at Ouedeme $\left(6^{\circ} 42^{\prime} 46^{\prime \prime} \mathrm{N}, 1^{\circ} 41^{\prime} 07^{\prime \prime} \mathrm{E}\right.$, altitude $21 \mathrm{~m}$ ), Benin. The experimental site is located in the Guinea savannah zone in Benin, with 986.3 and $1202 \mathrm{~mm}$ of total annual rainfall in 2010 and 2011, respectively. The genetic material used comprised 21 accessions of $O$. glaberrima selected for their origins from throughout West Africa and four $O$. sativa varieties as checks from an $O$. sativa core collection compiled by the National Institute of Agrobiological Sciences (NIAS), Japan (Kojima et al., 2005) (Table 1). Of these four checks, Nipponbare was known to have strong resistance to grain shattering (Konishi et al., 2006; Goto et al., 2009). The other three varieties were randomly selected from the remaining 68 varieties and there was no information in relation to their resistance to grain shattering.

The experiment was conducted during the wet season from June to December in 2010 and repeated in 2011. The site was in rainfed upland and no additional irrigation water was supplied during the experiments. Two treatments were made for the incidence of grain shattering. One treatment was to cover panicles on each hill by paper sacks at the heading stage to avoid grain shattering and the second one was a control without coverage by paper sack. An alpha-lattice design with three replications was adopted. The size of each plot was $1 \mathrm{~m} \times 2 \mathrm{~m}$ for each accession/variety and the plot was divided into two sub-plots $(1 \mathrm{~m} \times 1 \mathrm{~m}$ each): the nongrain shattering and control treatments. Hills were arranged by square planting $0.25 \mathrm{~m}$ apart. Three seeds were sown in each hill. All plots received compound N-P-K (15-15-15) fertilizer at the rate of $200 \mathrm{~kg} \mathrm{ha}^{-1}$ at 25 days after seeding (DAS) and urea (46\% nitrogen) at the rate of $50 \mathrm{~kg} \mathrm{ha}^{-1}$ at $45 \mathrm{DAS}$. The field was manually weeded regularly to maintain all plots weed-free and bird damage was avoided by hiring bird scarers during maturity. 
When $85 \%$ of grains matured, all hills except one border row were harvested. The samples were manually threshed and threshed paddy was air-dried. The paddy was winnowed manually and weighed. Grain moisture content was determined by moisture meter (AE 36093, Kett) and yield was expressed at $14 \%$ grain moisture content. Yield in the treatment to avoid grain shattering was considered as potential yield, while that in the control (no treatment to avoid grain shattering) gave control yield. Yield loss was therefore obtained by subtracting the control yield from the potential yield, and the percentage ratio of the lost yield to the potential yield was calculated for every accession/variety in each replication.

A linear mixed model procedure and a generalized linear mixed model procedure, both using a restricted maximum likelihood method (REML), were used to estimate the variance between the years. Further analysis was dependent on the interactive effect of Year $\times$ Genotype in the results of the REML; if the interactive Year $\times$ Genotype effect was significant, analysis of variance (ANOVA) was performed in each year separately. If not, ANOVA was done combining the results of the two years. Potential yield and control yield were subjected to the linear mixed model while percentage of lost yield by grain shattering to the potential yield was subjected to the generalized linear mixed model. To evaluate a difference between potential and control yields in each accession/variety, a ttest was used combining the data of the two years. Statistical analysis were performed using GenStat Discovery software (2003).

Table 1: Rice genotypes used and their origins.

\begin{tabular}{lcc}
\hline Accessions/Varieties & Species & Origins \\
\hline Nipponbare & O. sativa & Japan \\
Davao 1 & O. sativa & Philippines \\
Vary Futsi & O. sativa & Madagascar \\
Calotoc & O. sativa & Philippines \\
CG 14 & O. glaberrima & Senegal \\
CG 17 & O. glaberrima & Senegal \\
CG 20 & O. glaberrima & Senegal \\
Saliforeh & O. glaberrima & Sierra Leone \\
TOG 12303 & O. glaberrima & Cote d'Ivoire \\
TOG 5473 & O. glaberrima & Nigeria \\
TOG 5641 & O. glaberrima & Nigeria \\
TOG 5681 & O. glaberrima & Nigeria \\
TOG 5775 & O. glaberrima & Liberia \\
TOG 5829 & O. glaberrima & Liberia \\
TOG 6740 & O. glaberrima & Liberia \\
TOG 6748 & O. glaberrima & Liberia \\
TOG 6804 & O. glaberrima & Nigeria \\
TOG 7206 & O. glaberrima & Cote d'Ivoire \\
TOG 7420 & O. glaberrima & Sierra Leone \\
TOG 7442 & O. glaberrima & Nigeria \\
TOG 9066 & O. glaberrima & Nigeria \\
TOG 9276 & O. glaberrima & Nigeria \\
TOG 9280 & O. glaberrima & Nigeria \\
TOG 9281 & O. glaberrima & Nigeria \\
TOG 9300 & O. glaberrima & Nigeria \\
\hline
\end{tabular}




\section{RESULTS}

The linear mixed model indicated highly significant interaction for potential yield $(\mathrm{P}<0.001)$ and significant interaction for control yield $(\mathrm{P}=0.0267)$ between year and genotype (Year $\times$ Genotype) in both 2010 and 2011. The generalized linear mixed model also indicated a highly significant interaction for percentage of lost yield $(\mathrm{P}<0.001)$ between year and genotype (Year $x$ Genotype) in both 2010 and 2011. Therefore, a single factor ANOVA for potential yield, control yield or percentage of lost yield was adopted separately for each year. All data of potential and control yields and percentage of lost yield are shown in Table 2.

Potential yield was generally higher in 2011 than in 2010 for both O. glaberrima and $O$. sativa, probably due to higher precipitation in 2011. Correlation coefficient of percentage of lost yield (the check varieties were included) between 2010 and 2011 was 0.853 , which was highly significant $(P<0.001)$, so that the incidence of grain shattering seemed to be a varietal characteristic. There were highly significant varietal differences $(\mathrm{P}<$ 0.01 or $\mathrm{P}<0.001)$ in potential yield, control yield and percentage of lost yield in both 2010 and 2011.

The average of potential yield in $O$. glaberrima was $2.42 \mathrm{t} \mathrm{ha}^{-1}$ in 2010 and $3.65 \mathrm{t}$ $\mathrm{ha}^{-1}$ in 2011; these values are comparable to the average potential yield of the $O$. sativa checks $\left(2.99 \mathrm{t} \mathrm{ha}^{-1}\right.$ in 2010 and $3.49 \mathrm{t} \mathrm{ha}^{-1}$ in 2011). In particular, one $O$. glaberrima accession, Saliforeh, depicted the highest yield $\left(5.91 \mathrm{t} \mathrm{ha}^{-1}\right)$ in 2011 and the second highest $\left(4.09 \mathrm{t} \mathrm{ha}^{-1}\right)$ after Nipponbare $(O$. sativa) in 2010. In addition to that accession, six $O$. glaberrima accessions had yields of more than $4 \mathrm{t} \mathrm{ha}^{-1}$ under rainfed upland conditions in 2011. Therefore, if resistance to grain shattering is improved, (potential) yield of O. glaberrima will not be inferior to that of O. sativa.

In the $O$. sativa check varieties, potential and control yields were not significantly different in the t-test combining the two year results, and percentage of lost yield was $3.48 \%$ in 2010 and $21.12 \%$ in 2011 on average, suggesting the effect of grain shattering on yield is small but incidence of grain shattering was a little bit higher in the condition where yields of the checks were higher. In contrast, potential and control yields were significantly different in most $O$. glaberrima accession except two (TOG 12303 and TOG 9300). The percentage of lost yield by shattering in $O$. glaberrima was, on average, 55.84 and $66.67 \%$ in 2010 and 2011, respectively. These values are much higher than the averages for $O$. sativa; all accessions other than TOG 12303 and TOG 9300 had significantly higher values than the $O$. sativa variety (Calotoc), thereby showing the highest percentage of lost yield. It was confirmed that grain shattering is a characteristic generally observed in O. glaberrima. On the other hand, clear varietal differences were also observed in the percentage of lost yield in $O$. glaberrima: its range was $3.71-85.21 \%$ in 2010 and $15.53-87.49 \%$ in 2011. However, the coefficient of variations $(\mathrm{CVs})$ of the percentage of lost yield in O. glaberrima0.35 in 2010 and 0.25 in 2011-were low compared to those of $O$. sativa -0.48 in 2010 and 0.45 in 2011. The varietal difference of the percentage of lost yield in $O$. glaberrima could be small compared to $O$. sativa.

Two $O$. glaberrima accessions, TOG 12303 and TOG 9300, did not show a significant difference between potential and control yields. Over the two years TOG 12303 and TOG 9300 constantly had as low percentages of lost yield (3.71 and 6.36\% in 2010 , respectively; 15.53 and $27.18 \%$ in 2011 , respectively) as did the $O$. sativa check varieties (3.48\% in 2010 and $21.12 \%$ in 2011 , on average). Percentages of lost yield in these $O$. glaberrima accessions did not differ significantly from the percentage in the $O$. sativa check (Vary Futsi) possessing the lowest value. These two $O$. glaberrima accessions can be suitable genetic material to improve resistance to grain shattering in the intraspecific breeding of $O$. glaberrima. 
D. MONTCHO et al. /Int. J. Biol. Chem. Sci. 7(2): 535-543, 2013

Table 2: Potential yield by avoiding grain shattering, yield loss by grain shattering and percentage (\%) of lost yield to the potential yield in 2010 and 2011.

\begin{tabular}{|c|c|c|c|c|c|c|c|}
\hline \multirow{2}{*}{$\begin{array}{l}\text { Accessions } \\
\text { /varieties }\end{array}$} & \multicolumn{3}{|c|}{2010} & \multicolumn{3}{|c|}{2011} & \multirow[t]{2}{*}{ t-test } \\
\hline & $\begin{array}{c}\text { Potential } \\
\text { yield } \\
\left(\mathrm{t} \mathrm{ha} \mathbf{c}^{-1}\right)\end{array}$ & $\begin{array}{l}\text { Control yield } \\
\left(\mathrm{t} \mathrm{ha}^{-1}\right)\end{array}$ & $\begin{array}{c}\% \text { of lost } \\
\text { yield }\end{array}$ & $\begin{array}{l}\text { Potential yield } \\
\quad\left(\mathrm{t} \mathrm{ha}^{-1}\right)\end{array}$ & $\begin{array}{c}\text { Control } \\
\text { yield } \\
\left(\mathrm{t} \mathrm{ha}^{-1}\right)\end{array}$ & $\begin{array}{c}\% \text { of lost } \\
\text { yield }\end{array}$ & \\
\hline \multicolumn{8}{|c|}{ O. glaberrima } \\
\hline CG 14 & 2.53 & 0.98 & 60.75 & 3.87 & 1.43 & 62.57 & $* * *$ \\
\hline CG 17 & 3.42 & 2.05 & 40.42 & 6.66 & 1.16 & 67.55 & $* * *$ \\
\hline CG 20 & 1.98 & 0.68 & 64.41 & 3.86 & 1.59 & 64.14 & $* *$ \\
\hline Saliforeh & 4.09 & 2.15 & 46.95 & 5.91 & 2.32 & 62.52 & $* *$ \\
\hline TOG 12303 & 2.13 & 2.05 & 3.71 & 2.98 & 2.51 & 15.53 & $\mathrm{~ns}$ \\
\hline TOG 5473 & 1.66 & 0.53 & 68.04 & 2.39 & 0.31 & 87.49 & $* * *$ \\
\hline TOG 5641 & 3.34 & 0.50 & 85.21 & 2.80 & 0.68 & 76.66 & $* * *$ \\
\hline TOG 5681 & 2.30 & 1.04 & 58.40 & 4.69 & 1.97 & 62.98 & $*$ \\
\hline TOG 5775 & 2.45 & 1.17 & 52.09 & 3.81 & 1.10 & 71.92 & $* *$ \\
\hline TOG 5829 & 2.64 & 1.16 & 55.37 & 4.04 & 1.79 & 56.94 & $* *$ \\
\hline TOG 6740 & 2.80 & 1.10 & 60.72 & 2.82 & 0.83 & 70.88 & $* * *$ \\
\hline TOG 6748 & 2.98 & 1.05 & 64.93 & 4.55 & 1.31 & 70.74 & $* * *$ \\
\hline TOG 6804 & 1.60 & 0.32 & 81.29 & 4.52 & 1.08 & 77.09 & $*$ \\
\hline TOG 7206 & 2.30 & 0.74 & 70.47 & 4.70 & 1.89 & 64.96 & $*$ \\
\hline TOG 7420 & 3.19 & 1.37 & 57 & 2.79 & 0.77 & 72.82 & $* * *$ \\
\hline TOG 7442 & 1.56 & 0.45 & 73.13 & 3.09 & 0.36 & 82.87 & $* *$ \\
\hline TOG 9066 & 2.73 & 1.07 & 59.92 & 4.54 & 1.57 & 65.81 & $* *$ \\
\hline TOG 9276 & 1.63 & 0.36 & 77.32 & 3.48 & 0.77 & 84.88 & $* *$ \\
\hline TOG 9280 & 1.89 & 0.72 & 63.18 & 3.11 & 0.68 & 77.74 & $* * *$ \\
\hline
\end{tabular}


D. MONTCHO et al. / Int. J. Biol. Chem. Sci. 7(2): 535-543, 2013

\begin{tabular}{|c|c|c|c|c|c|c|c|}
\hline TOG 9281 & 1.83 & 0.62 & 65.90 & 3.29 & 0.74 & 78.77 & $* * *$ \\
\hline TOG 9300 & 1.72 & 1.61 & 6.36 & 1.73 & 1.14 & 27.18 & ns \\
\hline Average & 2.42 & 1.03 & 57.88 & 3.65 & 1.24 & 66.76 & \\
\hline $\mathrm{CV}$ & 0.28 & 0.52 & 0.35 & 0.25 & 0.48 & 0.25 & \\
\hline \multicolumn{8}{|l|}{ O. sativa } \\
\hline Nipponbare & 5.72 & 5.52 & 3.46 & 4.44 & 0.56 & 13.36 & ns \\
\hline Vary Futsi & 2.71 & 2.66 & 1.91 & 4.19 & 0.52 & 11.64 & ns \\
\hline Calotoc & 1.34 & 1.26 & 6.15 & 1.65 & 0.63 & 35.66 & ns \\
\hline Davao1 & 2.17 & 2.13 & 2.25 & 3.68 & 0.74 & 23.43 & ns \\
\hline Average & 2.99 & 2.89 & 3.48 & 3.49 & 0.61 & 21.12 & \\
\hline $\mathrm{CV}$ & 0.5 & 0.55 & 0.48 & 0.28 & 0.14 & 0.45 & \\
\hline Significance & $* * *$ & $* * *$ & $* * *$ & $* *$ & $* * *$ & $* * *$ & \\
\hline LSD & 0.99 & 0.53 & 13.30 & 1.49 & 1.24 & 17.24 & \\
\hline
\end{tabular}

LSD: Least Significant Difference; $\mathrm{t}$-test for each accession/variety as to whether the potential yield is significantly higher than the control yield combining the two years data 


\section{DISCUSSION}

Using the data on number of spikelets per unit ground area before shattering, Dingkuhn et al. (1998) have shown that potential yield of the $O$. glaberrima accession, CG 14, is comparable to that of a high yielding $O$. sativa variety, WAB56-104, and suggested that low yield of $O$. glaberrima is caused by grain shattering. This study, with the larger number of $O$. glaberrima accessions tested, confirmed that yield without grain shattering (potential yield) was not lower in $O$ glaberrima than in $O$. sativa.

Wild plant species demonstrate seed shattering, which is a useful trait to secure and disperse individuals of the next generation; in the process of domestication of wild species, grains that had reduced the shattering habit were selected to diminish grain yield loss by seed shattering (Zhou et al., 2012) and nonseed shattering is one of the most important characteristics in cultivated crops (Fuller, 2007). In this experiment, almost all $O$. glaberrima accessions tested were susceptive to grain shattering. The ease of grain shattering seems to be a character of $O$. glaberrima and this may come from its short history of domestication compared to $O$. sativa.

From observation of an abscission layer between the grain and the apical end of a pedicel during the maturing stages of $159 \mathrm{O}$. sativa varieties, Jin et al. (1982) have reported that the order of varieties for easiness of grain shattering is as follows: varieties possessing abscission layers cracked, varieties possessing abscission layers non-cracked and varieties possessing no abscission layers. In the evaluation of $42 \mathrm{O}$. glaberrima accessions, all accessions possessed abscission layers but some $O$. glaberrima accessions produced incomplete abscission layers, which cannot be seen in O. sativa (Jin et al., 1990); these accessions had some resistance to shattering compared to those with complete abscission layers but they are still classified as "easily shattering" (Jin et al., 1990). In this study, TOG 12303 showed the lowest values of the percentage of lost yield, which was $3.71 \%$ in 2010 and $15.53 \%$ in 2011 . These values are slightly not significantly higher than, but comparable to, those of Nipponbare, which has strong resistance to grain shattering (Konishi et al., 2006; Goto et al., 2009). Further morphological studies on the abscission layers of this accession and of TOG 9300 possessing similar values of percentage of lost yield with TOG 12303 will be necessary.

Nineteen of the $21 O$. glaberrima accessions tested were highly susceptive to grain shattering in the current study. These accessions would be expected to have complete abscission layers, but there was still a varietal difference within these 19 accessions the difference between the highest and lowest values was greater than the LSD (5\%) in both 2010 (44.79 vs. 13.30) and 2011 (30.55 vs. 17.24). Lodging is also a negative characteristic of $O$. glaberrima but there is a varietal difference within the species in the susceptibility to lodging (Futakuchi et al., 2008). The difference in lodging incidence might cause the varietal differences in the percentage of lost yield in these 19 accessions. Although $O$. glaberrima has been considered as low yield and this was a cause of its decreasing cultivation area in favor of $O$. sativa in Africa (Linares, 2002), the low yield could be the result of grain shattering, which can obviously be seen in this species. With grain shattering eliminated, potential yield is not low. Therefore, identification of genotypes to provide resistance to grain shattering is a crucial element to the intraspecific breeding of O. glaberrima, and two accessions, TOG 12303 and TOG 9300, possessing the requisite resistance were identified in this study.

\section{ACKNOWLEDGEMENTS}

This study was financially supported by the Ministry of Foreign Affairs, Japan and the Stress-tolerant Rice for Africa and South Asia Project funded by the Bill and Melinda Gates Foundation. The authors would like to acknowledge this support with deep thanks. 
The authors also thank the National Institute of Agrobiological Sciences (NIAS), Japan for providing rice accessions from the NIAS core collection.

\section{REFERENCES}

Coyne DL, Plowright RA, Fofana B. 1999. Observations on the susceptibility of Oryza sativa and resistance of Oryza glaberrima to the cyst nematode (Heterodra sacchari) and the influence of weed management in upland rice in ivory Coast. International Journal of Pest. Management, 45: 255-258.

Dingkuhn M, Jones MP, Johnson DE, Sow A. 1998. Growth and yield potential of Oryza sativa and $O$. glaberrima upland rice cultivars and their interspecific progenies. Field Crops Research, 57(1): 57-69.

Djedatin G, Ndjiondjop MN, Mathieu T, Cruz CMV, Sanni A, Ghesquière A, Verdier V. 2011. Evaluation of African cultivated rice Oryza glaberrima for resistance to bacterial blight. Plant Disease, 95(4): 441-447.

Fuller DQ. 2007. Contrasting patterns in crop domestication and domestication rates recent archeobotanical insights from the Old world. Ann. Bot., 100: 903-924.

Futakuchi K, Sie M. 2009. Better exploitation of African rice (Oryza glaberrima Steud.) in varietal development for resource-poor farmers in West and Central Africa. Agric J., 4: 96 -102.

Futakuchi K, Jones MP, Ishii R. 2001. Physiological and morphological mechanism of submergence resistance in African rice (Oryza glaberrima Steud.). Jpn. J. Tropic. Agr., 45: 8 -14.

Futakuchi K, Fofana M, Sié M. 2008. Varietal differences in lodging resistance of African Rice (O. glaberrima Steud.). Asian J. Plant Sci., 7: 569-573.

GenStat. 2003. GenStat for Windows. Release 4.23DE Discovery Edition. VSN International Ltd.: Hemel Hempstead, UK.
Ghesquiere A, Sequier J, Second G, Lorieux M. 1997. first step towards a rational use of African Rice ( $O$. glaberrima) in rice breeding through in a contig line concept. Euphytica, 96: 31-39.

Goto A, Sasahara H, Shigemune A, Miura K. 2009. Hokuriku 193: a new high-yielding indica rice cultivar bred in Japan. Japan Agricultural Research Quarterly, 43(1): $13-18$.

Jin ID, Terao H, Inouye J. 1982. On the cracking of abscission layer in Asian rice cultivar (Oryza sativa L.). Jpn. J. Crop Sci., 51: 542-545.

Jin ID, Inouye J, Quat NN. 1990. Histological peculiarities of the abscission layers of African rice, Oryza glaberrima Steud. and its relation with degree of grain shedding. Jpn. J. Crop Sci., 59: 475-480.

Jones M, Dingkuhn M, Aluko GK, Semon M. 1997. Interspecific Oryza Sativa L. x $O$. glaberrima Steud. progenies in upland rice improvement. Euphytica, 94(2): 237246.

Kawano N, Ito O, Sakagami JI. 2008. Flash flooding resistance of rice genotypes of Oryza sativa L., Oryza glaberrima Steud., and interspecific hybridization progeny. Environ. Exp. Bot., 63: 9-18.

Kojima Y, Ebana K, Fukuoka S, Nagamine T, Kawase M. 2005. Development of an RFLP-based Rice Diversity Research Set of Germplasm. Breeding Science, 55: 431 -440 .

Konishi S, Izawa T, Lin SY, Ebana K, Fukuta Y, Sasaki T, Yano M. 2006. An SNP caused loss of seed shattering during rice domestication. Science, 312: 1392 - 1396.

Linares OF. 2002. African rice (Oryza glaberrima): history and future potential. Proc. Natl. Acad. Sci., 99: 16360-16365.

Maji AT, Gana AS, Ukwungwu MN. 2010. Responses of Oryza glaberrima accessions to rice stresses and their morphological characteristics. Afr. J. General Agric., 6: 229 - 234.

Moukoumbi YD, Sie M, Vodouhe R, Bonou W, Toulou B, Ahanchede A. 2011. 
Screening of rice varieties for their weed competitiveness. African Journal of Agricultural Research, 6(24): 5446-5456.

Ndjiondjop MN, Manneh B, Cissoko M, Drame NK, Kakai-Glele R, Bocco R, Baimey H, Wopereis M. 2010. Drought resistance in an interspecific backcross population of rice (Oryza spp.) derived from the cross WAB56-104 (O. sativa $) \times$ CG14 (O. glaberrima). Plant Science, 179: 364-373.

PlowRight RA, Coyne DL, Nash P, Jones MP. 1999. Resistance to the rice nematodes Heterodera sacchari, Meloidogyne graminicola and $M$. incognita in Oryza glaberrima and $O$. glaberrima x $O$. sativa interspecific hybrids. Nematology, 1: 745752.

Sahrawat KL, Sika M. 2002. Comparative tolerance of $O$. sativa and $O$. glaberrima rice cultivars for iron toxicity in West Africa. Int. Rice Res. Notes, 27: 30-31.

Sie M, Dogbe YS, Coulibally M. 2005. Selection of interspecific hybrids $(O$. sativa $\mathrm{x} O$. glaberrima or lowland NERICAs) and intraspecifics adapted to rainfed lowland growing conditions. Int. Rice Comm. Newslett., 54: 47-51.

Thiémélé D, Boisnard A, Ndjiondjop MN, Chéron S, Séré Y, Aké S, Ghesquière A, Albar L. 2010. Identification of a second major resistance gene to Rice yellow mottle virus RYMV2 in the African cultivated rice species, $O$. glaberrima.
Theoretical and Applied Genetics, 121(1): 169-179.

Tobita S, Sahrawat KL, Diatta S, Jones MP. 2003. Response of African rice (Oryza glaberrima Steud.) to phosphate application in the upland of a P-deficient soil in the humid forest zone of West Africa. In Proceedings of the 2nd International Symposium for Phosphorus Dynamics in Soil-Plant Continuum. Perth, Western Australia, Australia, 21-26 September 2003, pp. 70-71.

Ukwungwu MN, Williams CT, Okhidievbie O. 1999. Screening of Africa rice, Oryza glaberrima Steud., for resistance to the African rice gall midge, Orseolia oryzivora Harris and Gagne. J. Food Technol. Africa 4: 108-110.

Vaughan DA, Morishima H, Kadowaki K. 2003. Diversity in the Oryza genus. Curr. Opin. Plant Biol., 6: 139-146.

Williams CT, Hidievbie O, Ukwungwu MN, Dakouo D, Nacro S, Hamadoun A, Kamara SI. 1999. Multilocational screening of $O$. sativa and $O$. glaberrima for resistance to African gall midge $O$. oryzovora. Int. Rice Res. Notes, 24: 26-27.

Zhou Y, Lu D, Li C, Luo J, Zhu BF, Zhu J, Shangguan Y, Wang Z, Sang T, Zhou B, Hana B. 2012. Genetic control of seed shattering in rice by the APETALA 2 transcription factor SHATTERING ABORTION 1. Plant Cell 24: 1034 -1048. 